\title{
EXTRACTION AND TRANSFORMATION OF IFC DATA TO CITYGML FORMAT
}

\author{
Mohammed Jawaluddeen Sani*1,2, Ivin Amri Musliman ${ }^{1}$, Alias Abdul Rahman ${ }^{1}$ \\ ${ }^{1}$ Department of Geoinformation, Universiti Teknologi Malaysia (UTM), Johor, Malaysia \\ mjsani71@gmail.com, alias.fksg@gmail.com, ivinamri@utm.my \\ ${ }^{2}$ Department of Surveying and Geoinformatics, Federal Polytechnic, Bauchi, Nigeria,
}

KEY WORDS: BIM, GIS, IFC, CityGML and Transformation

\begin{abstract}
:
The integration of Geographic Information System (GIS) and the Building Information Modeling (BIM) referred to as the merging of the two systems for the purpose of data interoperability. The need to share information between the two systems is what motivated the integration process purposely for geospatial analysis. This can be achieved through their data exchange formats such as; City Markup Language (CityGML) and Industry Foundation Classes (IFC). The formats are the two most prominent key schemas of GIS and BIM systems respectively. The integration is a step towards information exchange or sharing (data interoperability) between the two systems. The selection of the two most prominent data exchange formats is as a result of their widespread applications in the GIS and BIM domains. However, the differences in geometric and the semantics information hinders data interoperability (information sharing) between GIS and BIM. Also, coupled with the difference in schema structure and the level of information richness between IFC and CityGML., This paper, propose a geometry transformation process that can be used to extract and transform IFC building objects to that of CityGML building objects to enable 3D model design and constructed using BIM tool to be easily reused in 3D GIS applications which will be able to support the CityGML model format. Where the geometric information will be extracted using the IFC tree-structure (hierarchy) and transformed to destination CityGML.
\end{abstract}

\section{INTRODUCTION}

The world is currently looking at the possibilities of creating a more controllable, collaborative, seamless and visually realistic information management environment for Geographic Information System (GIS) and building industry. The integration of GIS and BIM data developed as a significant research area for the generation of valued information that could be of paramount importance for decision-making. Several efforts were made to harness the rich information in BIM and utilized them in GIS, but no established rule or standard to integrate the information elements between the two systems. Thus, a proper integration method at the data level is obviously essential. From the vantage point of GIS, there are many benefits related to using BIM information in its applications e.g. indoor service implementation, like emergency management (directing and finding evacuation paths in a fire situation) and indoor-outdoor relating services, like seamless navigation (Kang, 2018). Though, an undefine integration model of GIS and BIM method (s) may cause some problems, such as the difficulty for the user to forecast or expect the model integration results. These problems may likely cause the user to have second thought with regard to the use of the GIS and BIM model's integration information. In order to have a solution to these problems, the user should be able to define the integration process in a more homogenous technique (ISO TC211) from the viewpoint of use, therefore, the results should be undoubtedly foreseeable.

However, in some situation, the difficulty(s) in forecasting the model integration result is as a result of the challenges that are related to interoperability between GIS data and BIM data. This is coupled with the fact that the two systems of GIS and BIM are primarily designed for a different purposes (Amirebrahimi et al., 2016) and from different knowledge area (Kiavarz et al., 2018) with their own specialized functionalities, and have been therefore, the need to achieve data integration. used by different specialist over time. Also, the two systems of GIS and BIM have two different data exchange formats such as; City MarkUp language (CityGML) and Industry Foundation Classes (IFC) respectively. Therefore, it is challenging to come up or to develop any system that can sustain the two systems proficiencies (abilities) and provide a smooth integration of GIS and BIM. Consequently, a more feasible approach at this point is to achieve data integration between GIS and BIM. Nevertheless, the 3D building objects developed by the BIM systems and the geodata objects created and managed in the GIS systems are quite different in their representation approaches and data storage formats, their data integration still presents a challenging task. These challenges include the following; the difference in reference systems between BIM and GIS, Loss of semantics information, Geometric representation of 3D objects in BIM and GIS and harmonization of the LoDs. In this paper we tend to focus on the geometric data extraction and transformation for the GIS and BIM integration.

Amirebrahimi et al, (2016a) in their research proposed 3-group of GIS and BIM levels of integration, which include; Process level, application level, and data level as indicated in figure 1. At the process level, the two domains (BIM and GIS) are harness in a comprehensive workflow and collaborate, whereas at the application level new applications are developed to harness the relevant functionalities of the two systems (GIS and BIM), on another hand, the current applications are extended through plugins. Also, the authors identified stress at the data level, data structures are improved to change the needs of other application, sometimes it is the current data standards that are extended. The integration at the application level can only be achieved if there is a perfect flow of information between GIS and BIM at the data level, at this point the integration at the data level essential, 
The integration at the data level is basically referred to the transformation of geometry and semantic data between BIM and GIS, as earlier stated the aim of the transformation is to achieve data integration of the two systems. In this paper, we concentrated on the integration at the data level where we focus more on presenting a geometric transformation framework of
CityGML and IFC. The framework will include process of extracting the data (information) from IFC building model and also, the process of transforming the planar coordinates system PCS) to the real world coronates system (RWCS)

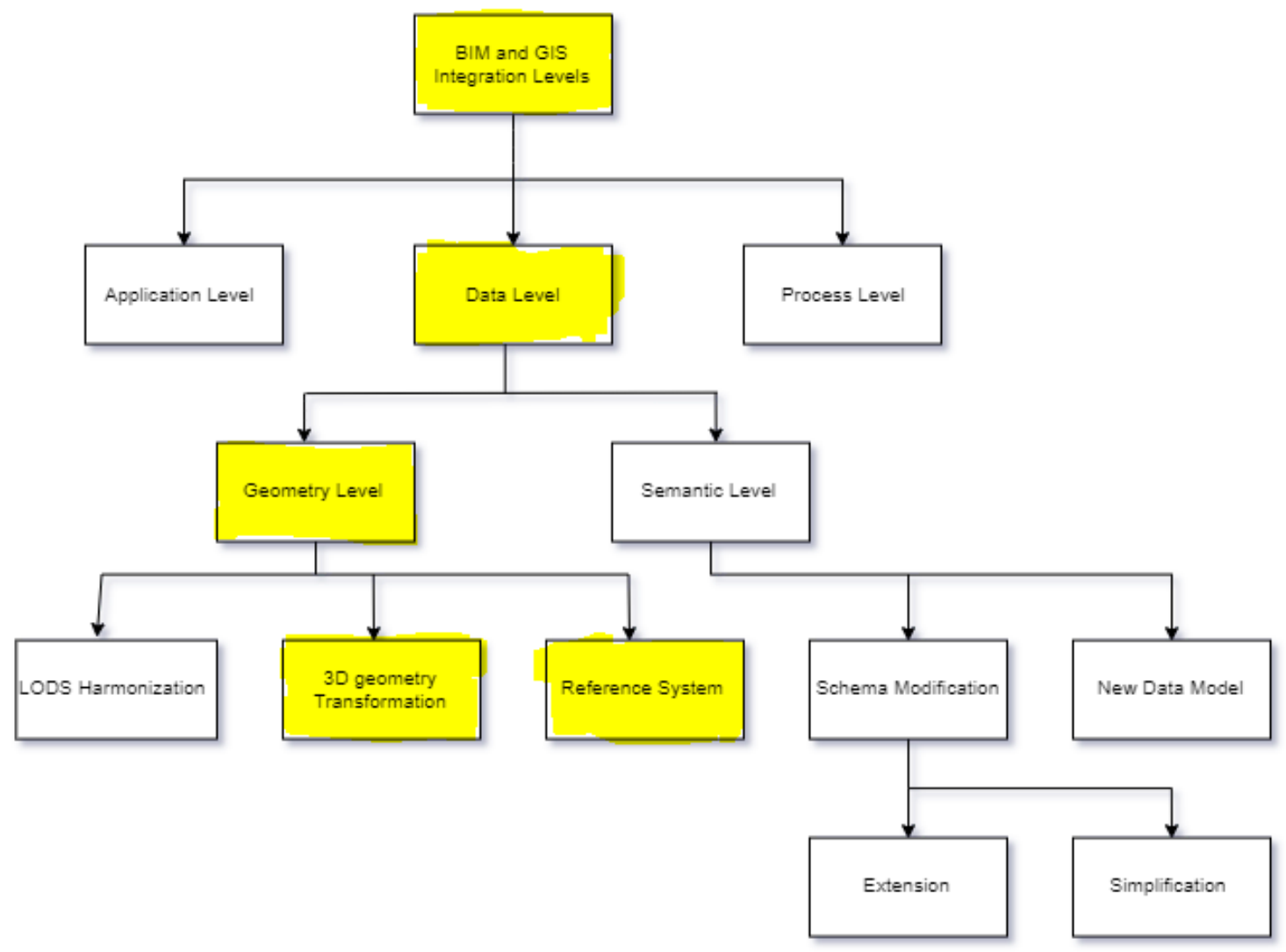

Figure 1. Levels of BIM and GIS integration.

The remainder of this paper is structured as follows: briefly introduces GIS and BIM data exchange formats (IFC and CityGML). Geometric and coordinates transformation. Presentation of the three methods of representing 3D geometry for both IFC and CityGML models. Presentation of the methodology for the extraction. Presentation of discussion, Conclusion and future work.

\subsection{Data Exchange Format}

The data exchange format is a strong part of the data level integration, the integration of GIS and BIM cannot be achieved without the data exchange format. Data format always change as a result of data interoperability between GIS and BIM. There are many data formats for storing 3D geometry among which are COLLADA, SHP, CityGML, GML, IFC, VRML, KML, and $\mathrm{X} 3 \mathrm{D}$ although the most important among them are: IFC and CityGML (Zhu et al., 2018).

\subsubsection{CityGML Data Format}

CityGML is developed by the Special Interest Group (SIG) of OGC group, it is an open standard data model designed to exchange and store 3D data models of 3D cities and landscapes based on GML in an XML format (Gröger et al., 2012). CityGML is an application schema for GML3 which is a standard for the exchange and sharing of 2D and 3D geospatial information over the internet (Yao et al., 2018). Also, the CityGML identifies the relevant attributes and distinctive relations of a 3D city. Yao et al presented that the CityGML is of great important for the simplification and maintenance of 3D city model (sustainable 3D city models) (Yao et al., 2018). Unlike IFC, CityGML represent 3D objects using only B-Rep.

Like IFC, CityGML model identifies building model in 5 Levels of Detail (LoDs) ranging from LoD0 being the lowest to LoD4 being the highest (i.e. LoD0, LoD1, LoD2, LoD3, and LoD4) as shown in Figure 2. The LoDs are define as follows; LoD0 is a footprint on 2D of the building model, LoD1 is an extrusion of the building footprint in form of a block in 3D with flat roof, while LoD2 is block model which has differentiated and CityGML building models represents building in 5 LODs but still, CityGML is less complete and mature as in BIM, even in LoD4 being the highest LODs for both models.

\subsubsection{IFC Data Format}

IFC is an open data schema used for the exchange of information within AEC domains in the AEC industry (Amirebrahimi et al., 2016a), it is an EXPRESS-based. Also, it is an international standard identified by ISO 10303-21 (STEPFile) which is developed by buildingSMART (formerly International Alliance for Interoperability) developed in 1994 (Mignard \& Nicolle, 2014). The primary purpose of developing 
the IFC is to find a solution to the information exchange issues in the AEC industry where the need for information is very vital (Zhu et al., 2018), the inefficiency of level of development or Details (LODs), method

of representing 3D objects (for instance, Constructive Solid Geometry (CSG), Sweep Solid (SS) and Building Representative (B-Rep), and IFC standard. IFC generate the 3D model using one or the combination of the three. As aforementioned, the American Institute of Architects (AIA) identified or classified IFC models into 5 categories based on the details they contain by Levels of Development (LODs), it uses LODs to show the amount of information contained in a particular model (Durão et al., 2019). The five LODs were defined as LOD100 to LOD500 in ascending order AIA. Also, in addition to LODs, a LOD350 is defined by the BIMForum working group (BIMFrorum).

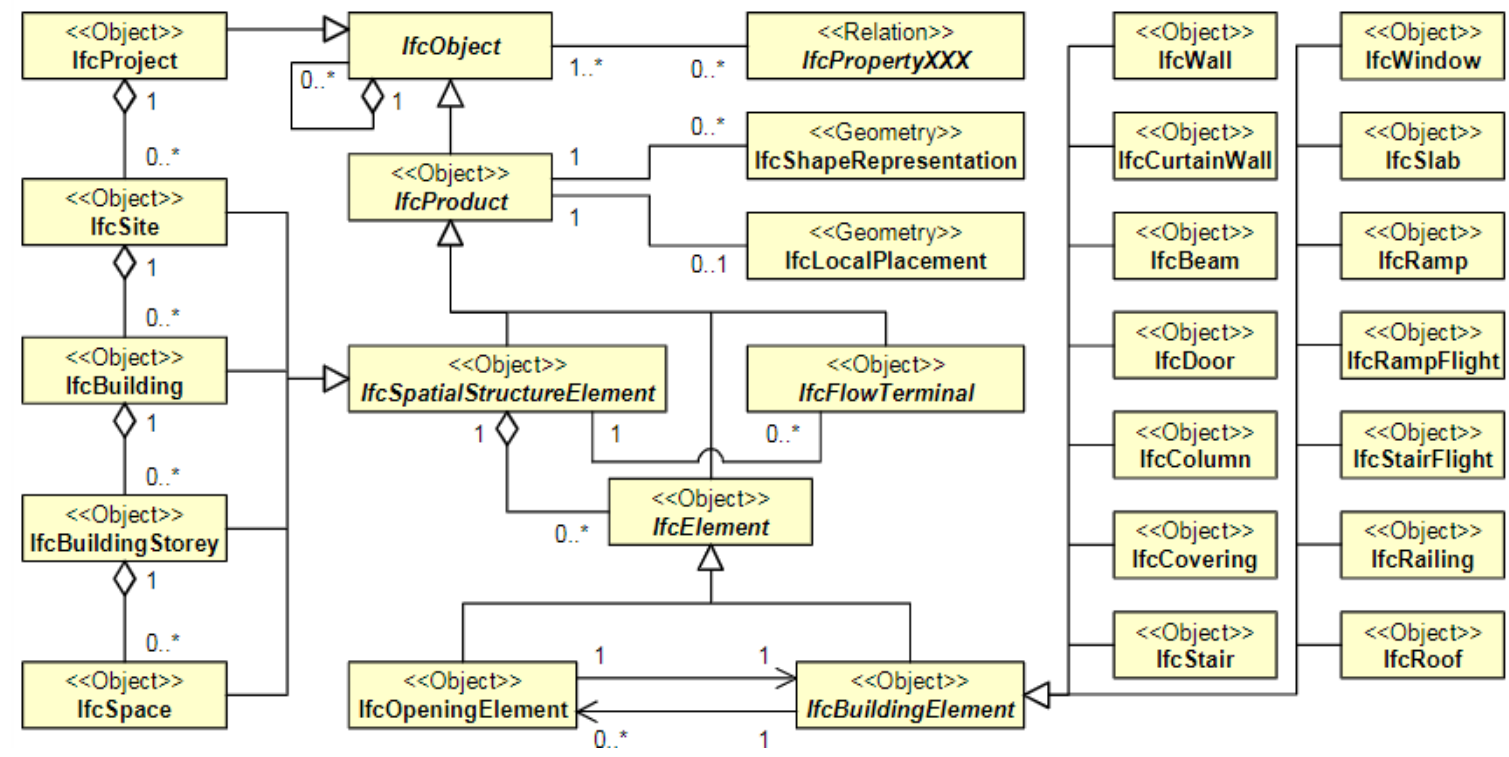

Figure 2. UML Diagram of some of the IFC Entities that can be extracted and used for CityGML model (Floros et al., 2017) Based on ISO 16739

\subsubsection{Relationship Between the Two Formats (Standards)}

The sharing of information between IFC and CityGML being standards of BIM and GIS is of Paramount importance do to the facts that it could solves problems like cost reduction which is also related to time-efficient management of projects, advanced data analysis and a unified view of the details of an area (El-Mekawy et al., 2012) identified some of the most relevant entities in IFC Models that can be used for geospatial analysis as shown in figure 2. Although, the complexities in the integration between the two models and the way they treat geometries and semantics in their schemas is as a result of their diversity. Bearing in mind, IFC model mostly concentrates on the building environment therefore provides a comprehensive information of structural elements like Doors, Windows, Walls, Roof, Floor etc. On the other hand, CityGML defines the Buildings as observed. It's also, defines and represent City Models which includes; Transportation objects, Water bodies, Buildings, Vegetations, etc (Nagel et al., 2009) in all the modules or city models aforementioned, IFC focuses only on buildings.

\subsection{IFC Building Model}

\subsubsection{Geometric Representation}

This paper concentrates on the representation of location and shape ifcLocalPlacement and ifcProductDefinitionShape respectively of IFC building Model elements. As mentioned in the previous section, three standards for representing geometry objects in $3 \mathrm{D}$ model were identified in IFC2 3 and IFC4 which is the most recent version of IFC, these includes CSG, $\mathrm{SS}$, and B-Rep. IFC uses one or the combination of the three to represents 3D objects. Unlike IFC, CityGML represents 3D objects using only B-Rep.SS uses a 2D profile to describe a 3D geometry coupled with its sweeping direction. CSG utilizes the outcome of a series of Boolean operations such as: Union, difference, and Intersection, and intersection of primitive objects to represent 3D model (object). These primitive objects could be cones, cylinder, pyramids etc. are important information for the creation of a model and stored in a GCS tree. Finally, B-rep represents a 3D object using its bounding surfaces and is mainly used for the representation of complex objects such as doors and windows.

\subsubsection{Geometric and Coordinates Transformation}

BIM and GIS integration could be operated on three levels, the application level, method level and data level (Amirebrahimi et al., 2016). The integration at data level is basically identified as the geometry transformation and semantic information between BIM and GIS domains, as stated in the previous section the objective is to achieve data interoperability between the two systems (Jusuf et al., 2017). Many researches on the integration of BIM and GIS were made. These researches focused on different application, process and method (Amirebrahimi et al., 2016) of BIM and GIS integrations. Most of the researches concentrated more on the semantics with few on the geometric aspect of the transformation which is vital for the integration of BIM and GIS. We propose a framework for the geometric and coordinates transformation of the IFC and CityGML building models. 


\section{METHODOLOGY}

\subsection{IFC Geometry}

Zhu et al, (2019) propose an Open Source approach for the transformation where the geometric information was extracted using spatial structure, but failed to use CityGML in their study LODs were neglected. The detailed geometric information about building can be extracted from designed and 3D BIM models using IFC as the data format for the building model, which could be automatically exchanged, there is need for a readily available framework for the transformation of the geometric data

In order to extract the geometric information of a building elements, two categories of information are important, and should be considered, these include: the placement of the element (IfcLocalPlacement) and its representation (IfcProductDefinitionShape). The placement defines the location of an element and the representation defines the shape of that element. Figure 1 below presents the attribute structure of an IFC geometry. The process comprises of the following;

First, as earlier stated the framework will basically relies on the ifcPlacement and the ifcProductDefinationshape of the building elements (ifcWindow, ifcDoor etc). The following were adopted for the geometric transformation process (as summarized):

The geometric data of the building objects in question was extracted from the IFC file.

Secondly, the local coordinates of the objects' vertices were computed using the following equation;

$$
\left(\begin{array}{l}
x^{b} \\
y^{\prime} \\
z^{s}
\end{array}\right)=A x\left(\begin{array}{l}
W x \\
W y \\
W z
\end{array}\right)+\left(\begin{array}{l}
x \\
y \\
z
\end{array}\right)
$$

Where:

$(\mathrm{Wx}, \mathrm{Wy}, \mathrm{Wz})$ :- represent the direction Vector of sweeping A: -represent the sweeping distance

Thirdly, is the computation and the transformation of the LPCS to RWCS using a transformation matrix equation as presented by (Wu \& Hsieh, 2007) in the final step, CityGML object Model is generated. The model can only be generated once the LPCS was successfully transformed to the GCS. The B-Rep will also be transformed from the SS.

\subsection{The Transformation Process}

This study presented a geometric transformation framework to simplify the IFC to CityGML transformation of selected building objects. The significant requirements for the transformation framework are identified as summarized:

i. After the transformation will let the user to choose the building objects from the IFC file in order to view and transform.

ii. The information of the IFC object selected will be displayed, like coordinate system, geometric data, object placement, etc. iii. The major IFC object (beams, column, floors etc.) will be automatically transformed into CityGML objects based on the designed framework.

\subsection{IFC Tree-Structure}

The IFC file structure (Tree-Structure) will be adopted for the geometry transformation in this study. IFC files create a building model based on pre-defined structure that builds the model in a logical way. When it is saved, the IFC file format classes are arrange in a hierarchical order or according to their types and based on the application intend to use the IFC model. The level is based on parent-child relationship in the order; ifcProject(Project) - ifcSite(Property)—ifcBuilding (Building) - ifcBuildingStorey(Floor)--

ifcBuildingElements(Object according to IFC classes). The IFC tree-structure is shown Figure 3.

The IFC tree-structure is an IFC tree in the form of an inverted tree structure where the root is the ifcProject as shown figure 3 below. In this study the IFC tree comprises of five levels from the ifcProject to ifcSite (ranging from ifcSite_1 to ifc_n) this is followed by ifcBuilding Storey(ranging from ifcBuildingStorey_1 to ifcBuildingStorey_n) the level is the ifcBuilding (ranging from ifcBuilding_1 to ifcBuilding_n) finally is the ifcBuildingElements (the building may have more than one elements such as: ifcWindow, ifcDoor, ifcRoof, ifcWall etc)

The spatial structure is primarily form by the elements in IFC. IFC being a component based model, where the information about an element can be extracted, parsed and processed disjointedly (Deng et al., 2016) for instance information about a window, these information include shape (ifcProductDefinationShape) and location (ifcLocalPlacement). Like IFC, CityGML is also a component-based model where the information about an entity can be extracted, parsed, processed disjointedly. The use of IFC Tree structure, will determined the possibility of reaching the other levels in the structure from the root. Logically, the use of this tree structure will allow for the extraction of all the IFC elements of any IFC building model.

In this study, the geometric information of 3D objects (Buildings) elements (ifcBuildingElement) can be essentially extracted based on the following; the element representation (ifcProductDefinitionShape) and its location (position) (ifcLocalPlacement). The two describes the shape and location of the object(element) respectively. Significantly, the two aforementioned attributes are required for the geometric information. In this framework, a successful extraction and transformation of geometric information will lead to a successful IFC and CityGML integration at the data level. And the goal of this framework is to achieve data interoperability between BIM and GIS systems. After the extraction the LPC from the IFC model using the IFC Treestructure in figure 4 . The next step is to determined position of 3D Building Models for CityGML as the following section. 


\section{DICUSSION}

\subsection{Rotation and Translation of A 3D Building Model}

In 3D graphics, every vertices or points of a model are defined using an $(x, y, z)$ of a plane. With an offset of $w$, the plane of the model can be placed anywhere in space, whereby the axis of the model in space is away from the origin $(0,0,0)$. The transformation of the vertex in space, or $3 \mathrm{D}$ object is given by the transformation $(4 \times 4)$ matrix multiply by the vector to give the resultant vertex position as shown in eqn. 1 .

3D Affine transformation matrix function will be used to transform and translate the object, that is needed to place the object in its right location (position) on the map. An affine transformation supports the polygon, or a line in space, therefore, supporting the translation, Rotation, and scaling of the object.

$$
\left(\begin{array}{l}
x \\
y \\
z
\end{array}\right)=\left(\begin{array}{ccc}
1 & 0 & 0 \\
0 & \cos \beta x & \sin \beta x \\
0 & -\sin \beta x & \cos \beta x
\end{array}\right)\left(\begin{array}{ccc}
\cos \beta y & 0 & -\sin \beta x \\
0 & 1 & 0 \\
\sin \beta x & 0 & \cos \beta x
\end{array}\right)\left(\begin{array}{ccc}
\cos \beta z & \sin \beta z & 0 \\
-\sin \beta z & \cos \beta z & 0 \\
0 & 0 & 1
\end{array}\right)\left(\begin{array}{l}
x^{\prime} \\
y^{\prime} \\
z^{\prime}
\end{array}\right)+\left(\begin{array}{l}
\Delta x \\
\Delta y \\
\Delta z
\end{array}\right)
$$

Where:

$(\mathrm{x}, \mathrm{y}, \mathrm{z})$ represent the Real World Coordinate System (RWCS)

$\left(x^{\prime}, y^{\prime}, z^{\prime}\right)$ represent the Local Planar Coordinate System (LPCS)

$(\Delta \mathrm{x}, \Delta \mathrm{y}, \Delta \mathrm{z})$ represent ranslation (change) from LPCS to RWCS from the origin

$(\mathrm{Bx}, \beta \mathrm{y}, \beta \mathrm{z})$ respectively represent the angle of rotation with respect to ( $\mathrm{x}$-axis, $\mathrm{y}$ - axis and $\mathrm{z}$-axis)

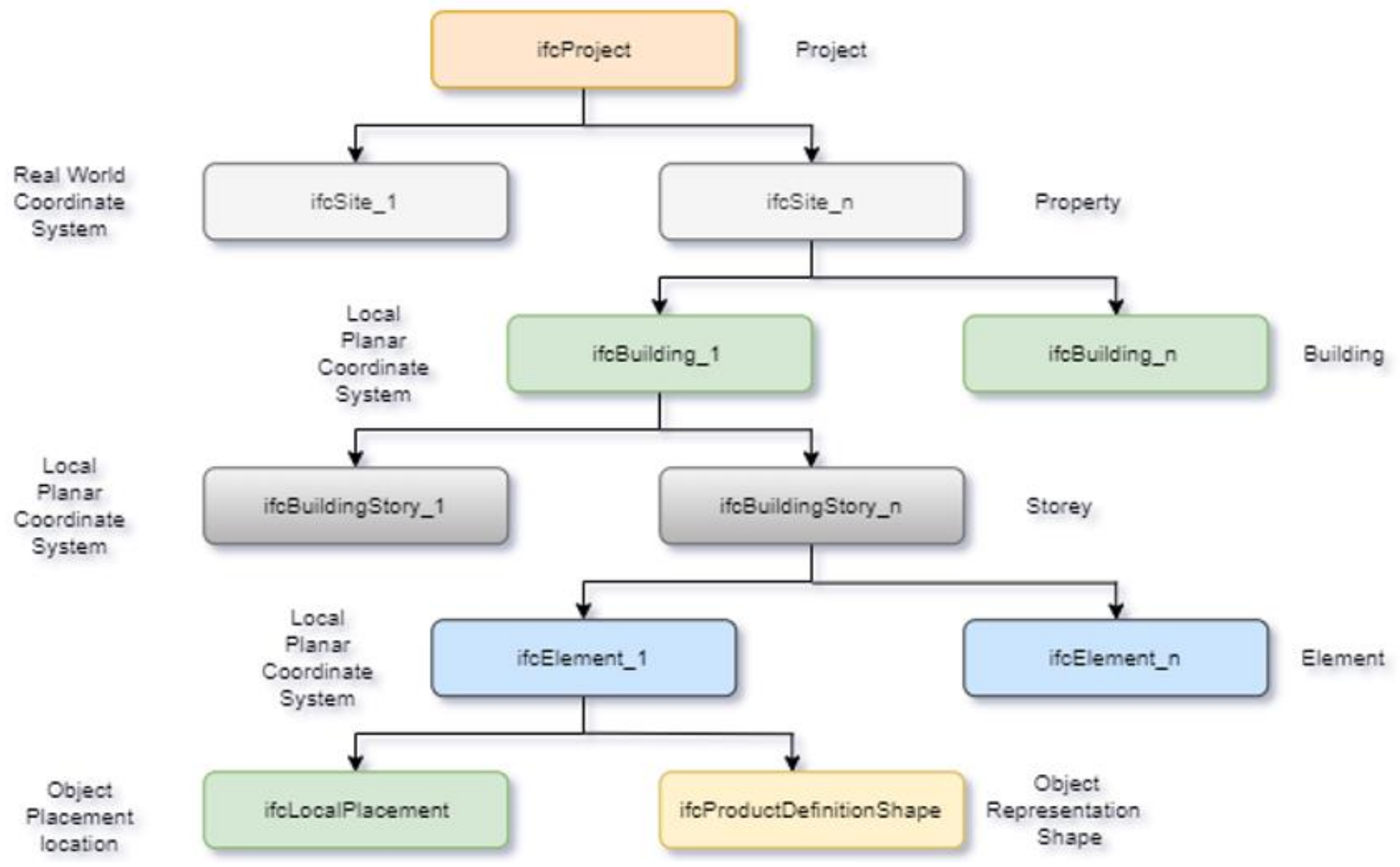

Figure 4. IFC Tree-Structure

\subsection{Geo-Coordinates Positioning for 3D models}

IFC files are 3D models in a planar surface, therefore do not contain geographic information. The files are often placed using the LPCS (CCS), mostly used in the computer 3D design tools, in this case the origin of the file is at $(0,0,0)$. RWCS (GCS), the RWCS is to define the location of object on the earth, considering the 3D spherical surface. The datum base on the spheroid, the angular unit of measure and the prime meridian were all included. However, the longitude and latitude used in the RWCS can locate any point on the earth's surface, the points are not uniform measure of distances, therefore it is only along the equator you find the distance represented by one degree of latitude equals to that one degree of longitude. Thus, different RWCS are strictly developed for different regions to stand for the representation of distance for the latitude and longitude.
The transformation of the 3D LPCS model to a 3D RWCS model in the host CityGML, is significant to carefully think and choose the right coordinate systems, so also the process for the translation of the coordinates to RWCS. At this point there is absolute need to select projected coordinates system, whereby, the locations of points on a curve Surface can be converted to the locations of the points on a flat plane. which is absolutely needed in the $3 \mathrm{D}$ coordinate projection. The coordinate projection supports the transformation tool to move the $3 \mathrm{D}$ models into the correct location on the map for various tools (i.e., IFC in LPCS to CityGML in RWCS) which can be verified easily using Google Earth based on world geodetic coordinate system (WGS 84). 


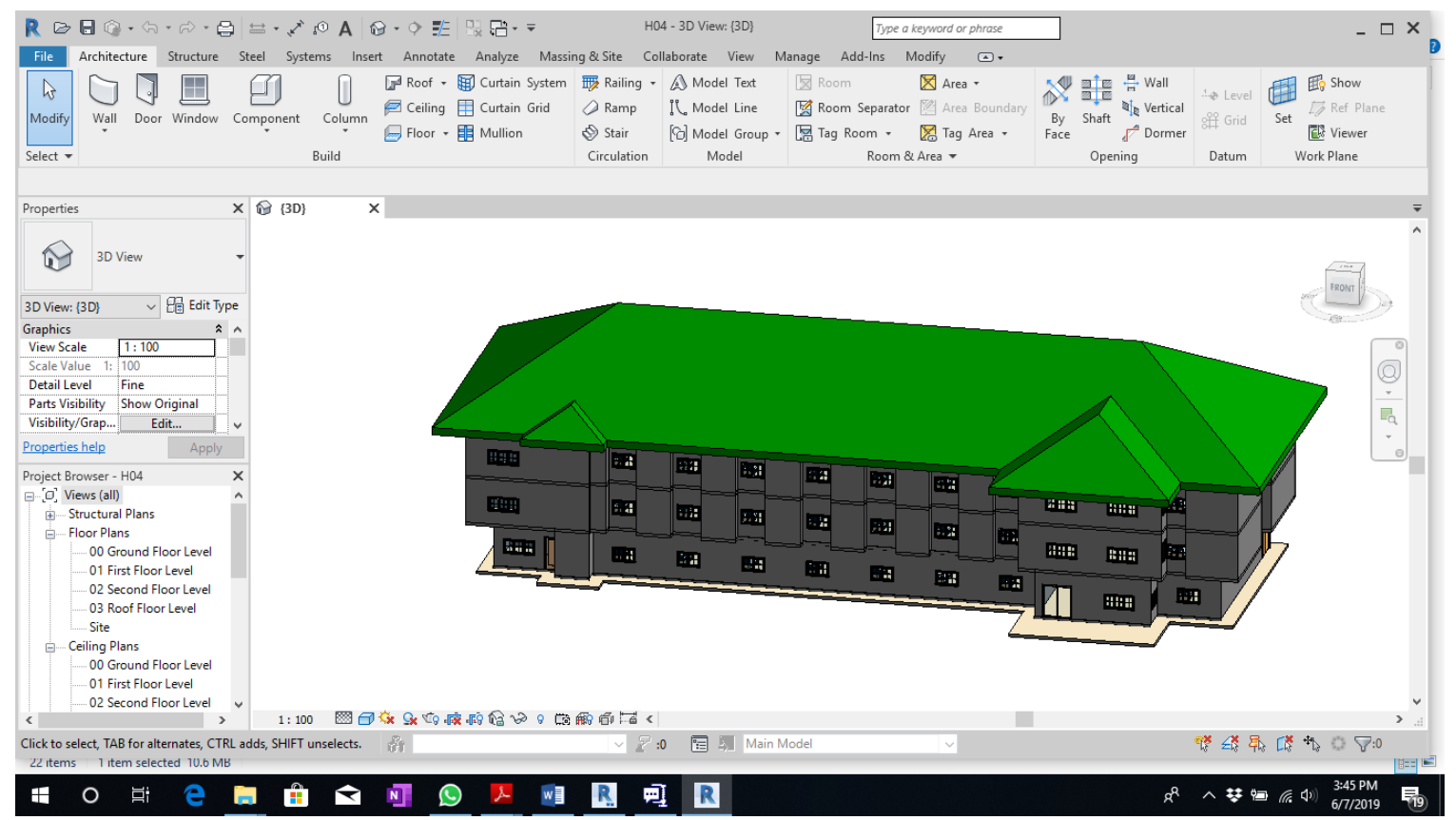

Figure 5. IFC building Model of Block HO4 Kolej Tun Fatimah of UTM Johor

Meanwhile, Both CityGML and IFC models are component based was the building is extracted base on elements such as Wall, Door, Roof, Window etc. The BIM models are graphically represented and detailed such as geometric information like size, volume, shape, height, orientation and non-geometric information such as quantity and performance data can be read directly from the models, as shown in Figure 5; the building model was developed using Autodesk Revit 2018 as a prototype for the transformation testing. Which is an apartment a block of building "block H02 Kolej Tun Fatimah" a hostel at UTM Johor. The transformation process was carried out by first converting the building model in _rvt file to IFC file. The IFC model was later exported to Sketchup where the model was transformed to CityGML building model and is visualized by FZK viewer as showed in figure 6 .

As aforementioned this paper concentrated mainly on the geometric level of the integration of CityGML and IFC, most precisely on the extraction of the building elements and transformation of the IFC building model to the destination CityGML building model.

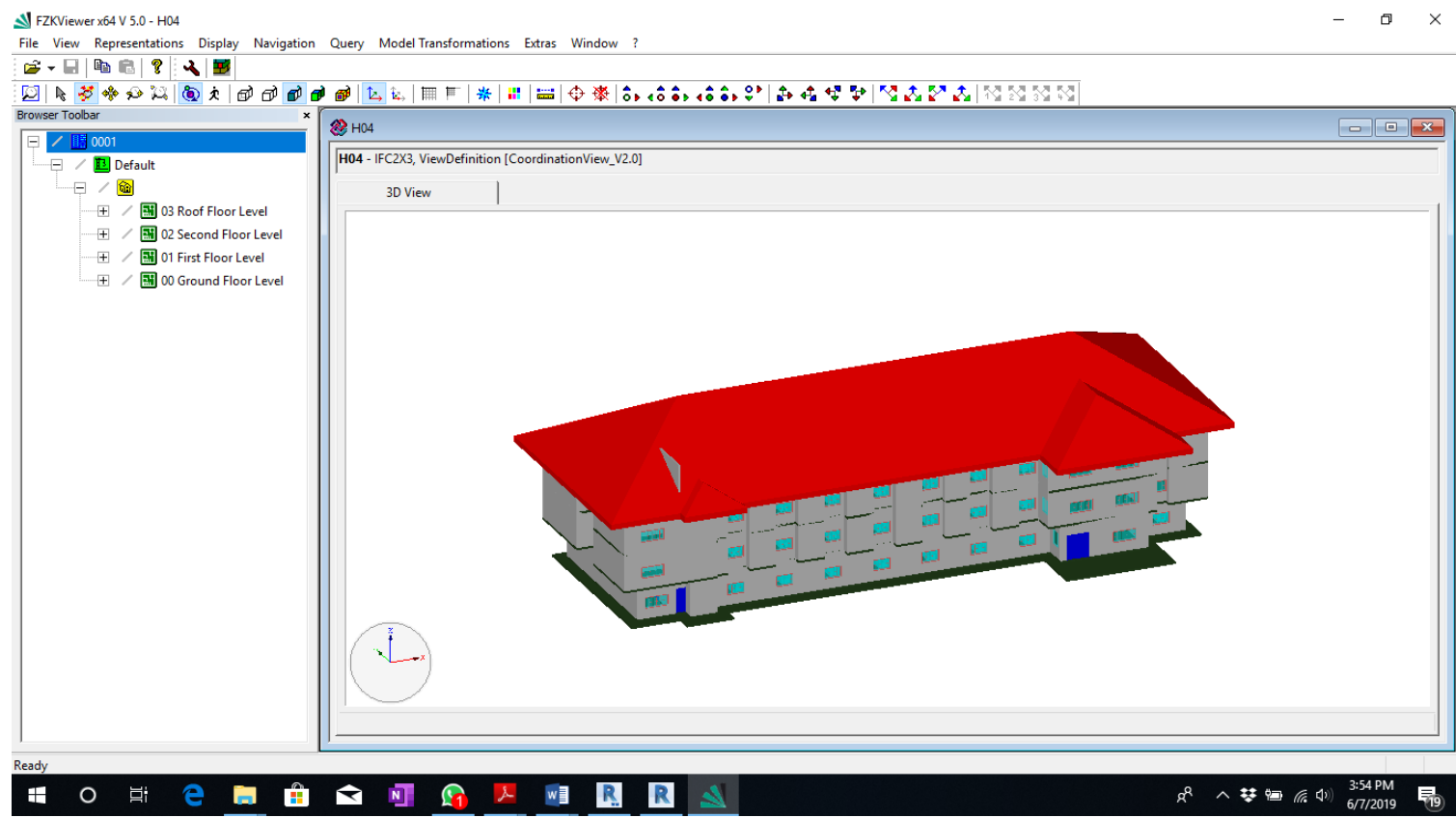

Figure 6. Transformed CityGML building Model of Block HO4 kolej Tun Fatimah of UTM Johor 


\section{CONCLUSION AND FUTURE WORK}

We propose a transformation process that can be used to extract and transform IFC building objects to that of CityGML building objects to enable 3D models design and constructed using BIM tools to be easily reused in 3D GIS applications which will be able to support the CityGML model format.

The proposed transformation methodology is achievable and suitable for transforming foremost building components of regular shapes to CityGML(B-Rep) from the IFC (SS) or (CSG) building models in order to support 3D GIS environment in reality for design, planning, management of information, and finally for decision making. It could be used in future to develop a user-friendly tool for the extraction and transformation of building elements from the IFC model to be used in CityGML building model with minimum human intervention.

The essence is for the building models to be transformed from IFC to CityGML for smooth exchange of information (Interoperability) between the two models. Though transforming more IFC objects with more complex SS geometry to CityGML model could be implemented, the process and the methodology for the extraction of the building elements and transformation of the building model proved to have practical values for 3DGIS applications.

\section{ACKNOWLEDGEMENT}

The authors would like to thank all the 3D GIS lab group members (UTM) for their contribution in one way or the other and the management of UTM in general. Also, we Will like to thank the management of Federal Polytechnic, Bauchi and Federal Ministry of Education Nigeria.

\section{REFERENCES}

Amirebrahimi, S., Rajabifard, A., Mendis, P., \& Ngo, T. 2016. A framework for a microscale flood damage assessment and visualization for a building using BIM-GIS integration. International Journal of Digital Earth, 9(4), 363-386. https://doi.org/10.1080/17538947.2015.1034201

Deng, Y., Cheng, J. C. P., \& Anumba, C. 2016. Mapping between BIM and 3D GIS in different levels of detail using schema mediation and instance comparison. Automation in Construction, 67, 1-21. https://doi.org/10.1016/j.autcon.2016.03.006

Durão, V., Costa, A. A., Silvestre, J. D., Mateus, R., \& de Brito, J. 2019. Integration of environmental life cycle information in BIM objects according with the level of development. IOP Conference Series: Earth and Environmental Science, $225, \quad 012075$. https://doi.org/10.1088/1755-1315/225/1/012075

El-Mekawy, M., Östman, A., \& Hijazi, I. 2012. A Unified Building Model for 3D Urban GIS. ISPRS International Journal of Geo-Information, 1(3), 120-145. https://doi.org/10.3390/ijgi1020120

Floros, G., Pispidikis, I., \& Dimopoulou, E. 2017. Investigating integration capabilities between IFC and citygml lod3 for 3D city modelling. International Archives of the
Photogrammetry, Remote Sensing and Spatial Information Sciences - ISPRS Archives, 42(4W7), 1-6. https://doi.org/10.5194/isprs-archives-XLII-4-W7-1-2017

Gröger, G., Kolbe, T., Nagel, C., \& Häfele, K.-H. 2012. OGC City Geography Markup Language (CityGML) Encoding Standard. Ogc 12-019, 1-344. https://doi.org/OGC 12-019

Jusuf, S., Mousseau, B., Godfroid, G., \& Soh, J. 2017. Path to an Integrated Modelling between IFC and CityGML for Neighborhood Scale Modelling. Urban Science, 1(3), 25. https://doi.org/10.3390/urbansci1030025

Kang, T. 2018. Development of a Conceptual Mapping Standard to Link Building and Geospatial Information. ISPRS International Journal of Geo-Information, 7(5), 162. https://doi.org/10.3390/ijgi7050162

Kiavarz, H., Jadidi, M., Rajabifard, A., Sohn, G., Vi, C., \& Vi, W. G. 2018. BIM-GIS ORIENTED INTELLIGENT KNOWLEDGE DISCOVERY. XLII(October), 1-2.

Nagel, C., Stadler, A., \& Kolbe, T. H. 2009. Conceptual Requirements for the Automatic Reconstruction of Building Information Models from Uninterpreted 3D Models. Proceedings of the International Archives of Photogrammetry, Remote Sensing and Spatial Information Sciences, 46-53. https://doi.org/10.1.1.150.9821

Wu, I. C., \& Hsieh, S. H. 2007. Transformation from IFC data model to GML data model: Methodology and tool development. Journal of the Chinese Institute of Engineers, Transactions of the Chinese Institute of Engineers,Series A/Chung-Kuo Kung Ch'eng Hsuch K'an, 30(6), 1085-1090. https://doi.org/10.1080/02533839.2007.9671335

Yao, Z., Nagel, C., Kunde, F., Hudra, G., Willkomm, P., Donaubauer, A., ... Kolbe, T. H. 2018. 3DCityDB - a 3D geodatabase solution for the management, analysis, and visualization of semantic 3D city models based on CityGML. Open Geospatial Data, Software and Standards, 3(1). https://doi.org/10.1186/s40965-018-0046-7

Revised August 2019 